\title{
DESARROLLO DE UN ENTORNO VIRTUAL DE APRENDIZAJE PARA CENTROS DE CAPACITACIÓN TÉCNICA EN BOGOTÁ D.C.
}

\author{
Virtual learning environment develop \\ for technical training centers in Bogotá D.C.
}

DIEGO ALEJANDRO DURAN ESPITIA

vcorreal@sanmateo.edu.co

VÍCTOR JAVIER CORREAL ÁNGEL

daduran@sanmateo.edu.co

ALBERTO MORALES OSPINA (Docente investigador)

amoraleso@sanmateo.edu.co

Semillero Gestión en las Organizaciones

Fundación Universitaria San Mateo (Colombia)

Artículo de investigación formativa

Recepción: 29 de octubre de 2020

Aceptación: 12 de abril de 2021 


\title{
Resumen:
}

La educación a distancia fue la pionera de la inclusión social en la educación. Con ella las distancias entre clases sociales, distinciones de género, de etnia y raza se abolieron; es un gran paso en la búsqueda de la igualdad. De allí nace la educación virtual; no solo coadyuvo a romper paradigmas, también traspasó fronteras. Ya no hay lugar del territorio donde no pueda llegar la educación. La virtualidad ha globalizado la educación y generó una explosión de conocimiento mundial. En pro de esta alternativa educativa, se desarrollan procesos de inclusión de centros de educación tradicionales. Estos pueden proyectar su visión basados en las nuevas tecnologías, con visión de empresa, incursionando en los entornos virtuales de aprendizaje. Para ello se desarrollan procesos de investigación de tipo mixto, recolectando datos y formulando propuestas; así como diseñando procedimientos a usar. Lo anterior, con el fin de lograr desarrollar entornos virtuales de aprendizaje para los centros de educación técnica que adelantan su labor educativa en la ciudad de Bogotá D.C., lugar donde se encuentra el objeto de investigación y donde se desarrolla la prueba piloto de la propuesta.

Palabras clave: educación a distancia; ambiente educacional; aprendizaje en línea.

\begin{abstract}
:
Distance education was the pioneer of social inclusion in education. With it the distances between social classes, gender, ethnic and racial distinctions were abolished; it is a great step in the search for equality. From there the virtual education is born; Not only did it help break paradigms, it also crossed borders. There is no longer a place in the territory where you cannot get to education. Virtuality has globalized education and generated an explosion of world knowledge. In favor of this educational alternative, processes of inclusion of traditional education centers are developed. They can project their vision based on new technologies, with a business vision, venturing into virtual learning environments. For this, mixed-type research processes are developed, collecting data and formulating proposals; as well as designing procedures to use. The foregoing, in order to develop virtual learning environments for technical education centers that carry out their educational work in the city of Bogotá D.C., where the research object is located and where the pilot test of the proposal is developed.
\end{abstract}

Keywords: distance education; educational environment; e-learning. 


\section{Introducción}

La educación ha tenido grandes desarrollos en el acompañamiento que realiza a las distintas civilizaciones. Cabe anotar, de manera contemporánea cómo ha cobrado gran protagonismo en la vida diaria de los pueblos. La tecnología es base fundamental en este desarrollo. Por ello, se torna indispensable investigar a fondo la potencialización que pueden dar las Tecnologías de Información y Comunicación (TIC) a las capacidades de la educación en su papel de gestor de desarrollo de la sociedad. Entonces, el Estado en su relación con la nación y desde la óptica de las ciencias sociales; gesta como agente generador y facilitador de dicho desarrollo tecnológico.

En ese sentido, desarrollar estrategias de implementación de la TIC en el modelo educativo tiene un papel prioritario; basta con ver las necesidades que desnudó la pandemia del COVID-19 a nivel mundial. En el ámbito educativo el agente que generó las posibilidades de continuar con los procesos fue la tecnología, sin llegar a afirmar que fue efectiva en su totalidad; realmente nadie estaba preparado para tan funestos eventos. Por ello, es importante considerar que la tecnología será parte de los modelos educativos; además, se conformará como herramienta del proceso pedagógico. Asimismo, los docentes dentro de sus procedimientos didácticos están llamados a usarlos de manera cotidiana.

De igual manera, el modelo educativo debe transformarse, adecuarse y sincronizarse con las nuevas tecnologías. Los documentos rectores dentro de los distintos modelos deben ser adecuados de tal manera que tanto instituciones y educandos tengan las reglas claras sobre cómo se maneja la educación con metodologías que incluyan herramientas tecnológicas; bien sea un método de educación virtual o el modelo de educación remota. Con ello el Estado tiene una gran oportunidad de llegar a todo el territorio nacional, aplicando la teoría del control institucional a cambio del control territorial.

La finalidad de la investigación está en generar una propuesta de implementación de proyectos educativos para los centros de educación técnica en la ciudad de Bogotá. Lo anterior, ocasionado por un contexto socioeconómico donde estos centros son parte importante en el modelo educativo de la ciudad. Es una manera rápida y efectiva de incorporarse al mundo laborar y mantener aun las posibilidades de continuar hacia la educación superior (Garzón, Rojas y Acosta, 2019; Cortés, 2019).

\section{Metodología}

Durante el proceso se desarrolla una investigación con finalidad aplicada; centrada en la resolución de problemas en un contexto de la educación virtual. Busca la aplicación o utilización de conocimientos, desde varias áreas especializadas, con el propósito de implementarlos de forma práctica. Tiene por objetivo resolver el acceso a la educación ofrecida por los centros de capacitación técnica de la ciudad de Bogotá. 
El enfoque es mixto, al utilizar los métodos cualitativo y cuantitativo se logra que se complementen entre sí. Esto hace que las desventajas de cada método se contrarresten y los resultados que se obtengan sean más satisfactorios. En la investigación se necesita evitar ambigüedades y respuestas imparciales; por ejemplo, una metodología cualitativa puede ser contraproducente. Al aplicar un método mixto, se minimiza el margen de error, contrarrestando las respuestas obtenidas del método cualitativo con aquellas obtenidas cuantitativamente.

Ahora bien, en el desarrollo de la investigación se utilizaron instrumentos de una manera mucho más versátil que el de una investigación de un solo método. Combinar elementos de ambos tipos de investigaciones permite crear encuestas y entrevistas con respuestas más elaboradas, las cuales sirvan para obtener resultados mixtos; así como una revisión documental e investigación de campo. Es decir, los instrumentos mixtos permiten a la investigación combinar las herramientas para que los resultados produzcan respuestas mucho más eficientes.

Por consiguiente, la investigación se validó en tres (3) ámbitos. Uno es la validación de contenido, basados en la obtención de la información de fuentes científicas serias. Un segundo ámbito es la validación de criterios, con lo cual se usarán estándares externos que brindan una referencia clara de los resultados. El tercer ámbito es la validación del constructo, en la cual las categorías de la investigación serán valoradas dentro las teorías que conforman la investigación. Esto garantiza un resultado ajustado a procesos cognitivos comprobados de manera científica.

El universo poblacional está en los centros de capacitación técnica que tiene la ciudad de Bogotá; conforman una población bastante amplia en la ciudad. Estos centros se han dedicado a la educación presencial y no han logrado crecer en su proyecto de empresa. Por tanto, esta población será la gran beneficiada con los resultados de esta investigación.

\section{Resultados y discusión}

En Europa, más específicamente en España, se manejan factores de nacimiento de la educación a distancia. Relacionan este evento desde el mismo nacimiento de la escritura, pasando por la revolución industrial; tomando en cuenta la creación del correo y la transmisión de mensajes; así, sobrepasando el tiempo y el espacio. García (1999) destaca la óptica con que este país ve la educación a distancia y su nacimiento con la siguiente afirmación: "Con la aparición de la escritura se propiciaba el que otros entendiesen un mensaje que una persona distante en el espacio y/o el tiempo, había escrito" (p. 2).

Macmillan Education Iberia crea una plataforma de educación virtual llamada Plexus. Basa su desarrollo en las competencias docentes, concepto muy contemporáneo; desde la óptica de la tecnología y del talento humano. La asociación didáctica ofrece cursos online dirigidos a docentes y sus capacidades en electrónica y mecánica; es una educación dirigida hacia la robótica. 
En consecuencia, a las dos (2) plataformas anteriores está Doqua la cual está dirigida a capacitar el personal en el manejo de herramientas tecnológicas. Busca nivelar los participantes en los conocimientos necesarios para cumplir labores virtuales en educación. Por último, en el ámbito internacional está Femxa; ofrece gran variedad de cursos, categorizados por áreas de conocimiento y horas de capacitación. Además, se cuenta con la homologación del Estado español y apoyo gubernamental en todo Europa.

De igual manera, Colombia ha desarrollado un sistema de educación virtual basado en la empresa privada con apoyo estatal. Paralelamente, cuenta con una oferta del mismo Estado, como tal, tiene el Servicio Nacional de Aprendizaje (SENA). Este ha sido referente de gran calidad en la educación a distancia y, con la transformación a educación virtual, el Ministerio de Educación Nacional apoya esta labor.

También se creó la Universidad Nacional Abierta y A Distancia (UNAD). Es la versión virtual de la Universidad Nacional de Colombia; funciona de manera autónoma e independiente con gran acogida en la sociedad. En el departamento de Santander, la Universidad de Bucaramanga fue pionera en la educación a distancia y es de las primeras en homologar títulos académicos obtenidos de manera virtual. Otro referente que ha logrado posicionarse con esta modalidad es la Universidad de San Buenaventura la cual ha implementado cursos de carácter no formal, homologados por el ministerio de educación.

De manera local, las diferentes instituciones de educación virtual tienen protagonismo de un punto más informal. Están las instituciones de idiomas como lo es Berlitz y el Centro Colombo Americano que han desarrollado sistemas presenciales. Sin embargo, desde tiempo atrás versificaron su actividad desde las plataformas digitales, creando ambientes virtuales de aprendizaje para enseñar un segundo idioma.

Otra manera local que existe, homologada por la secretaria distrital de educación, es el sistema MOOC. Este implementa diferentes instituciones de educación. Con dicho formato se facilita el proceso de enseñanza y es muy participativo e incluyente.

Para entender el proceso que se pretende, es necesario ahondar en las teorías de la educación. No se puede permitir que los medios tecnológicos sean los que rijan un proceso educativo. Con ello se da a entender que las teorías de la educación son fundamentales en el estudio.

La teoría conductista se relaciona, desde el punto de vista de la propuesta de educación virtual, como el aprendizaje de procedimientos nuevos. Abril et al. (2011) aducen que los teóricos le niegan la posibilidad de desarrollo a los procesos cognitivos porque no se pueden medir. No obstante, los comportamientos adquiridos para el manejo de las herramientas tecnológicas necesarias en este proyecto sí tienen un método para su medición. 
Aquí es donde esta teoría cobra valor dentro del articulo y se tiene en cuenta para el desarrollo de uno de los objetivos específicos, el cual asume la responsabilidad de implementar las herramientas necesarias para alcanzar el objetivo general.

En otro aspecto se tiene en cuenta el constructivismo, basados en el compromiso que se adquiere por parte de todos los actores en el proceso educativo. Araya, Alfaro y Andonegui (2007) consideran que es un compromiso donde el conocimiento se construye en mutua colaboración. El estudiante deja a un lado su posición pasiva y se convierte en actor activo en el proceso educativo que se adelanta. De esta manera, hace del conocimiento no un insumo, sino el resultado de procesos pedagógicos de vanguardia.

Para principios de la década de 1990, en Colombia se implementa un modelo educativo el cual genera cambios sustanciales en la manera de desarrollar la educación en el país. Se trató de la Ley 30 de 1992, entre muchas cosas, genera el espacio para la educación a distancia y la educación virtual. Mena et al. (2008) comentan el amplio rango de la ley en la normatividad de toda América Latina y el Caribe; así como los vacíos que presenta en su jurisprudencia.

Más específico aún, en su artículo 15 esta ley dice textualmente: "Las instituciones de Educación Superior podrán adelantar programas en la metodología de educación abierta y a distancia, de conformidad con la presenteLey" (Ley 30 de 1992). Por tanto, se nota la falta de compromiso del Estado en fortalecer y apoyar la virtualidad como parte del modelo educativo. De manera más contemporánea, se logra visualizar la importancia e impacto que tiene.

Los diferentes Gobiernos han implementado varios esquemas de educación. Asimismo lo realizan los entes locales; en la capital de la República los últimos Gobiernos han optado por fortalecer la educación desde una visión más social. Con ello, fortalecieron infraestructura en todo nivel, de manera integral; dotaron con herramientas tanto para educación presencial como para virtual. Por consiguiente, se logra una mayor inclusión de los ciudadanos en los programas del Estado.

La educación virtual hace parte del desarrollo progresivo de la educación a distancia. Cedeño (2019) consideraban que la concepción que tiene el estudiante sobre el entorno virtual es vital en el desarrollo del proceso de formación. Es así como en todo el mundo se vivieron épocas de desigualdad de oportunidades, no por falta de intención de los Estados, sino por falta de capacidades.

De ahí la necesidad de educar la sociedad, en pro del desarrollo de la nación. La educación a distancia es la estrategia que surge como alternativa viable para poder cerrar las brechas en el aspecto de la educación. Además, como consecuencia también se cierran brechas sociales y económicas. 
Colombia no es ajena a este tipo de dificultades. En búsqueda de alternativas encuentra que en otros países la educación a distancia ha dado buenos resultados; así, se incorpora esta metodología dentro del modelo educativo del país. Mondéjar, Mondéjar y Vargas (2006), consentían la idea de que los entornos virtuales ayudaban a la creación de nuevas comunidades de aprendizaje, logrando alcanzar rincones alejados del territorio. Sin embargo, aún quedaban vacíos; aún había personas que no alcanzaban a estar dentro del radio de impacto de la estrategia. Esto se logra mitigar usando la tecnología; cuando nace la educación virtual, el margen de personas que no tienen acceso a la educación se reduce considerablemente.

Bogotá ha sido el mejor objeto de investigación para el país. A mediados del siglo XIX, la migración que se presenta desde todas las regiones de Colombia (a razón de la violencia) desnudan una cruel realidad. El migrante campesino que estaba llegando a la ciudad carecía de educación. El Estado no había llegado con todo su poder a cada colombiano. El centralismo se veía en todo su esplendor, donde se olvidaron de las regiones circundantes a la capital. De allí se inicia a buscar alternativas de educación para cada colombiano, sin importar donde estuviera; con ello la educación a distancia y, de manera más contemporánea, la educación virtual.

En las localidades de la capital se ha dado nacimiento a diferentes propuestas de educación. Una de ellas es la educación técnica la cual en poco tiempo preparan al estudiante en las competencias necesarias para el trabajo. Están enfocados de manera específica con una actividad en especial, de acuerdo con las necesidades del estudiante. Estas instituciones aún no logran la cobertura suficiente; la mayoría están en la educación presencial.

Orostica Verdugo (2020) afirma que no solo en las etapas superiores de la educación se puede incursionar en los entornos virtuales de aprendizaje, sino incluso en los niveles básicos. Por ello, es importante que esta investigación coadyuve al proceso de estas instituciones en la educación virtual, como forma de labor social. Así, empoderando al ciudadano de la participación relevante que tiene en la construcción del conocimiento y fortalecimiento de su propia educación. Esta investigación se fortalece en el ámbito social cuando se cierran brechas de desigualdad de oportunidades y mitigación del riesgo de analfabetismos en la ciudad.

\section{Conclusiones}

La ciudad de Bogotá necesita plantear un modelo de educación acorde con las necesidades de la ciudad. Además, basados en su contexto socio económico, en busca de no perder el ritmo de desarrollo y mantener la oferta laborar en un mercado cada vez más exigente. Con esto se consolida un proceso que debió tener mayor fortaleza desde décadas anteriores. 
Ahora bien, los Estados están llamados a gestionar las políticas públicas en el ámbito de la educación. Aquellas que reglamenten los nuevos procesos y proporcionen el acceso de los educandos a dichos procesos. De esta manera, el Estado se promueve como el ente facilitador de los procesos educativos en Colombia, específicamente, en la capital de la república.

Es necesario promover proyectos de educación virtual, los cuales coadyuben en el proceso de desarrollo educativo de una sociedad. En el contexto actual necesita distintas opciones de continuar en su desarrollo educativo. En consecuencia, el modelo de desarrollo económico del país se fortalece y garantiza el mantenimiento del estilo de vida de los bogotanos, de allí que, De la Ossa, (2009) manifestó de manera enfática que la virtualidad es el futuro de la educación en grandes urbes y se refería de manera especial en Bogotá. D.C., con ello se intenta generar estrategias que coadyuven a la administración distrital en generar igualdad de oportunidades de acceso a la educación. 


\section{Referencias bibliográficas}

Abril, D., Bichara, R., Lara, A., Jiménez, F. y Vidal, I. (2011). Conductismo y constructivismo ¿qué modelo usar? Revista Académica, (40), 8-31. Recuperado de http://repositorio.ucm. cl/bitstream/handle/ucm/1624/ abril_d_conductivismo.pdf?sequence $=1$ \&isAllowed $=y$

Araya, V., Alfaro, M., y Andonegui, M. (2007). Constructivismo: Orígenes y perspectivas. Laurus, 13(24), 76-92. Recuperado de https://www.redalyc. org/pdf/761/76111485004.pdf

Cedeño, E. (2019). Entornos virtuales de aprendizaje y su rol innovador en el proceso de enseñanza. Rehuso, 4(1), 119-127. Recuperado de: https://revistas.utm.edu.ec/index. php/Rehuso/article/view/1888

Cortés, J. (Comp.) (2019). La administración de empresas, una perspectiva desde el profesional mateísta hacia el futuro. Bogotá: Editorial Universitaria San Mateo

De la Ossa, H. (2009). Sentido TIC en la educación de Bogotá. Aula Urbana, (74), 6-7. Recuperado a partir de https://revistas.idep.edu.co/index. $\mathrm{php} / \mathrm{mau} /$ article/view/579

García, L. (1999). Historia de la Educación a Distancia. Revista Iberoamericana de Educación a Distancia, 2(1), pp. 8-27. https://doi.org/10.5944/ ried.2.1.2084
Garzón Daza, C., Rojas Ávila, L., y Acosta Triviño, G. (2019). Prospectiva de la competencias en la disciplina contable. En Doris Ordy Ruiz. (Comp.) Prospectiva del desarrollo de competencias para los profesionales de contaduría pública desde la perspectiva formación por ciclos propedéuticos (pp. 8-27). Bogotá: Editorial Universitaria San Mateo.

Ley 30 de 1992. Por la cual se organiza el servicio público de la educación superior. Diciembre 28 de 1992, Congreso de la República de colombia.

Mena, M., Rama, C. y Facundo, A. (2008). El Marco regulatorio de la Educación Superior a Distancia en América Latina y el Caribe. Bogotá: UNAD. Recuperado de https:// virtualeduca.org/documentos/observatorio/oevalc_2008_(marco. reg).pdf

Mondéjar, J., Mondéjar, J. y Vargas, M. (2006). Implantación de la metodología e-learning en la docencia universitaria: una experiencia a través del proyecto Campus Virtual. Revista Latinoamericana de Tecnología Educativa, 5(1), 59-71. Recuperado de http://www.unex.es/didactica/RELATEC/sumario_5_1.htm

Orostica Verdugo, K. V. (2020). Entorno Virtual de Aprendizaje: Campus UVM ONLINE. Universitas Tarraconensis. Revista de Ciències de l'Educació, 1(1). DOI: https://doi.org/10.17345/ ute.2020.1.2533 